\title{
Structural and abnormal electrical properties of excess PbO-doped lead lanthanum titanate thin films
}

\author{
Zhitang Song $\dagger$, Wei Ren $\ddagger$, Shixin Wang§, Lumin Wang\$, \\ Chenglu Lin $\dagger$, Liangyin Zhang $\neq$ and Xi Yao
}

$\uparrow$ State Key Laboratory of Functional Materials for Informatics

Shanghai Institute of Metallurgy, Chinese Academy of Sciences, Shanghai 200050,

People's Republic of China

\$ Electronic Material Research Laboratory, Xi' an Jiaotong University, Xi' an 710049,

People's Republic of China

$\S$ Department of Nuclear Engineering and Radiological Science, College of Engineering,

The University of Michigan, Ann Arbor, MI 48109, USA

Received 14 July 1999, in final form 14 October 1999

\begin{abstract}
Lead lanthanum titanate (PLT) thin films with excess PbO (from 0 to $20 \mathrm{~mol} \%$ ) were prepared by a metal-organic decomposition process. The ferroelectric properties and current-voltage $(C-V)$ characteristics of PLT films were investigated as a function of the excess $\mathrm{PbO}$. Abnormal ferroelectric and $C-V$ properties were observed in PLT films with excess $\mathrm{PbO}$. The polarization against applied electric field $(P-E)$ hysteresis loops were pinched before saturation of polarization of the films, and $C-V$ curves had four peaks instead of the two peaks found in the normal $C-V$ curves. The abnormal level of the hysteresis loops and $C-V$ curves deteriorate with increasing concentrations of excess $\mathrm{PbO}$ in the films. Electron probe microanalysis has revealed that there is excess Pb in PLT thin films. Auger electron spectroscopy has detected that the $\mathrm{Pb}$ accumulates at the interfaces between the thin film and the bottom electrode. Meanwhile, transmission electron microscopy has found that $\mathrm{PbO}$ nanocrystals on the interface between the PLT thin film and the bottom electrode, and clusters of vacancies and interstitials, in particular, exist in the PLT grains. Therefore, a part of the excess $\mathrm{PbO}$ may accumulate at the domain wall of the grains and the grain boundaries and the interface between the bottom electrode and film during the thermal annealing process of the films. Meanwhile, the oxygen vacancies of the grains will increase with the increasing concentration of the excess $\mathrm{PbO}$ in the films. The excess $\mathrm{PbO}$ and oxygen vacancies act as pinning centres and have a strong pinning effect on the domains. When the poling voltage is not large enough, part of the domains can overcome the force of the pinning, and abnormal ferroelectric and $C-V$ properties were observed.
\end{abstract}

(Some figures in this article appear in colour in the electronic version; see www.iop.org)

\section{Introduction}

Lead lanthanum titanate (PLT) is one of the important ferroelectric materials. It has excellent dielectric, ferroelectric, pyroelectric and electro-optic properties. PLT thin films have been widely studied for applications in dynamic random access memories (DRAM), uncooling infrared detectors and electro-optic devices [1-4]. Several methods such as sol-gel, metallo-organic decomposition (MOD), sputtering, laser ablation and evaporation techniques have been adopted to prepare the PLT thin films [5-9].

Because $\mathrm{Pb}$ is a volatile element in the $\mathrm{Pb}$-based perovskite system, some of $\mathrm{Pb}$ will volatilize during film thermal process and cause a $\mathrm{Pb}$ deficit in the films. Many researchers have added excess $\mathrm{PbO}$ during synthesizing the PLT films to compensate for the $\mathrm{Pb}$ deficit and to promote the formation of the perovskite phase of the films. Tuttle et al [10] studied the effect of addition of excess $\mathrm{Pb}$ on the formation of the perovskite phase by using a sol-gel process. They showed that excess $\mathrm{Pb}$ additions (up to $5 \mathrm{~mol} \%$ ) and fast heating rates increased the perovskite content and improved the ferroelectric properties of the films fired at $600{ }^{\circ} \mathrm{C}$. Bernstein et al $[11,12]$ also studied the effect of adding excess $\mathrm{Pb}$, by sol-gel and reactive rf magnetron sputtering methods. Even though the lowering of crystallization temperature to $550{ }^{\circ} \mathrm{C}$ was achieved with the addition of 9-16 mol\%, excess $\mathrm{Pb}$ in PZT thin films, the electrical properties were not very good. In particular, additions of excess $\mathrm{Pb}$ resulted in leaky films with poor hysteresis loop [11, 12]. Khan and Desu [13] studied the PLT films ( $28 \mathrm{~mol} \% \mathrm{La}$ ) with different excess amounts of $\mathrm{PbO}$. Their results showed that the PLT films with 5-10 mol\% of excess $\mathrm{PbO}$ had low dielectric loss and leakage current and high remnant polarization. Lefevre et al [14] used several different methods, such as excess $\mathrm{PbO}$ in the precursor solution, a $\mathrm{PbO}$ atmosphere during the film thermal treatment and a $\mathrm{PbO}$ overcoat on the surface of the 
ferroelectric films, to compensate for $\mathrm{Pb}$ deficiency and to eliminate fluorite formation in the PZT thin films. However, the effects of the excess $\mathrm{PbO}$ in the ferroelectric films on the structure and properties, especially on the electric properties at the electric fields which are smaller than the switching threshold are worth further investigation. Kim et al [15] studied the low-temperature growth of PZT thin films though the increase of excess $\mathrm{Pb}(50 \mathrm{~mol} \%)$ in a PZT ceramic target by using the rf magnetron sputtering method. The in situ heating temperature was as low as $520^{\circ} \mathrm{C}$ when depositing PZT thin films without any non-perovskite phase.

In this paper, the PLT thin films with a La content of $0-20 \mathrm{~mol} \%$ were prepared by the MOD process. The microstructures and electrical properties of the PLT thin films were investigated as a function of excess $\mathrm{PbO}$. Based on the experimental results, the abnormal phenomenon in the PLT thin films with excess $\mathrm{PbO}$ were tentatively explained by a ferroelectric domain pinning effect that was caused by $\mathrm{PbO}$ and oxygen vacancy pinning centres.

\section{Experimental}

The PLT thin films were prepared on silicon wafers by the MOD process. The PLT films were synthesized according to the formula $\mathrm{Pb}_{1-x} \mathrm{La}_{x} \mathrm{Ti}_{1-x / 4} \mathrm{O}_{3}$ with $x=0.1$ (abbreviation PLT10). The different amounts of excess $\mathrm{PbO}$ in the range of 0-20 mol\% were added to the PLT10 films to study effect of excess $\mathrm{PbO}$. The excess percentage of $\mathrm{PbO}$ in the A-sites is compared with $(1-x) \mathrm{Pb}$ in A-sites. The 0.5 M PLT precursor solution was prepared from lead acetate trihydrate, titanium n-butoxide and lanthanum nitrate. 2-butoxyethanol was chosen as a solvent. The details of the preparation procedure of the precursor solutions can be seen in [7].

Thermally oxidized (111) Si wafers were used as the substrates. Pt and Ti with different thickness were sputtered on Si wafers as the bottom electrodes. The precursor solutions were spin coated onto the substrates in a clean room. The 'wet' films were thermally treated at $180^{\circ} \mathrm{C}$ and $400{ }^{\circ} \mathrm{C}$ for $30 \mathrm{~min}$, respectively, at a heating rate of $3{ }^{\circ} \mathrm{C} \mathrm{min}{ }^{-1}$, the coating and heating processes were repeated eight times and the final films were heated to the temperature above crystallization at a heating rate of $1{ }^{\circ} \mathrm{C} \mathrm{min}{ }^{-1}$. The total thickness of the PLT films obtained in the above thermal treatments were about $1 \mu \mathrm{m}$.

The thermal analysis of the precursor solutions was carried out by TA2000 thermal analysis system. The structures and morphologies of the PLT films were analysed by a Rigaku D/MAX2000 x-ray diffractometer (XRD) with $\mathrm{Cu} \mathrm{K} \alpha$, atomic force microscopy (AFM), and highresolution transmission electron microscopy (HRTEM). The HRTEM was performed with a JEOL 4000EX electron microscope operating at $400 \mathrm{kV}$. The analytical TEM (AEM) was conducted with a JEOL 2000FX electron microscope operating at $200 \mathrm{kV}$ with a TN5500 energy dispersive $\mathrm{x}$-ray spectroscopy attachment. The composition of the films were analysed by a JEOL JCXA-733 electron probe x-ray microanalyser (EPMA) operating at a voltage of $15 \mathrm{kV}$ and a current of $20 \mathrm{nA}$. The incident electron beam was about $3 \mu \mathrm{m}$ in diameter. A crystal wavelength dispersive spectrometer was used for x-ray wavelength and intensity

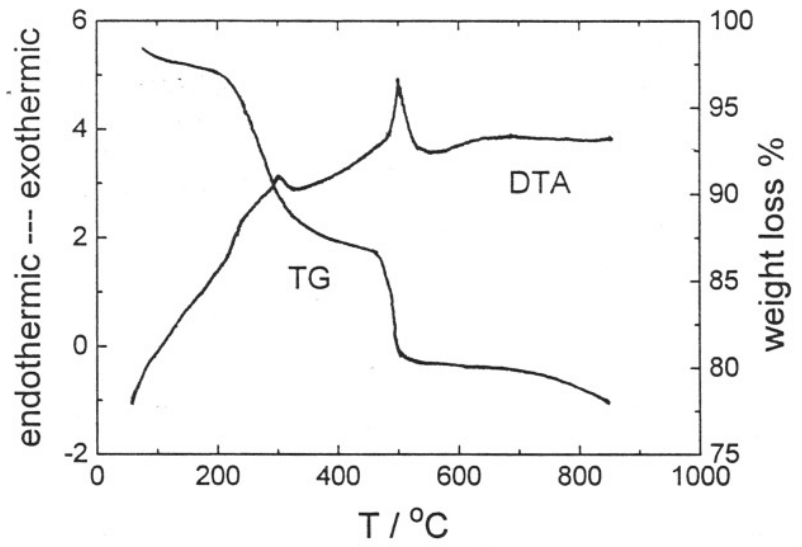

Figure 1. DTA and TG curves of the PLT10 precursor solution with $10 \mathrm{~mol} \%$ excess $\mathrm{PbO}$.

analysis. Three spots in each film sample were chosen in the measurement. Each element for the analysis in the thin films was calibrated by using a standard sample. The measured results were modified by the ZAF (atomic number correction factor, $\mathrm{Z}$, absorption correction factor, A, characteristic fluorescence factor, F) correction. A PerlinElmer PHI600 auger electron spectroscopy (AES) was used to investigate AES depth profile of the PLT10 films. The incident electron beam with an operating voltage of $3 \mathrm{kV}$, a current of $1 \mu \mathrm{A}$ and a beam diameter of $0.1 \mu \mathrm{m}$ was used. The Argon beam with an energy of $3 \mathrm{keV}$, a current of $6 \mu \mathrm{A}$ and a beam diameter of $2 \mathrm{~mm}$ was adopted to etch the film samples. For the electric measurements, Au top electrode (100 nm thick) with a diameter of $1 \mathrm{~mm}$ was deposited by dc sputtering. The ferroelectric polarization against applied electric field $(P-E)$ hysteresis loops were measured by a modified Sawyer-Tower circuit at a frequency of $1 \mathrm{kHz}$. The current-voltage $(C-V)$ curves were measured by HP4192A LF impedance analyser.

\section{Results}

\subsection{Thermal analysis}

Differential thermal analysis (DTA) and thermogravimetry (TG) curves of the PLT10 precursor solution with $10 \mathrm{~mol} \%$ excess $\mathrm{PbO}$ were shown in figure 1 . The solutions were dried at $120^{\circ} \mathrm{C}$ and then they were measured. In the TG curve it can be seen that there were four weight losses during the heating process. The weight loss at $135^{\circ} \mathrm{C}$ corresponded to the evaporation of the organic solvent. The decomposition of lead acetate led to the weight loss at $300^{\circ} \mathrm{C}$ and $(\mathrm{PbO})_{\mathrm{t}}$ was formed $[12,13]$. The weight loss at $450^{\circ} \mathrm{C}$ was caused by the decomposition of other organic compounds. At the high temperature of $750^{\circ} \mathrm{C}$, the evaporation of $\mathrm{Pb}$ became severe. At temperatures below $750^{\circ} \mathrm{C}$, there was no obvious loss of $\mathrm{PbO}$. Other PLT precursor solutions had similar results exhibiting four weight losses.

\subsection{Structures and morphologies}

The microstructures and morphologies of the PLT films are strongly affected by the precursor solution, substrate, 


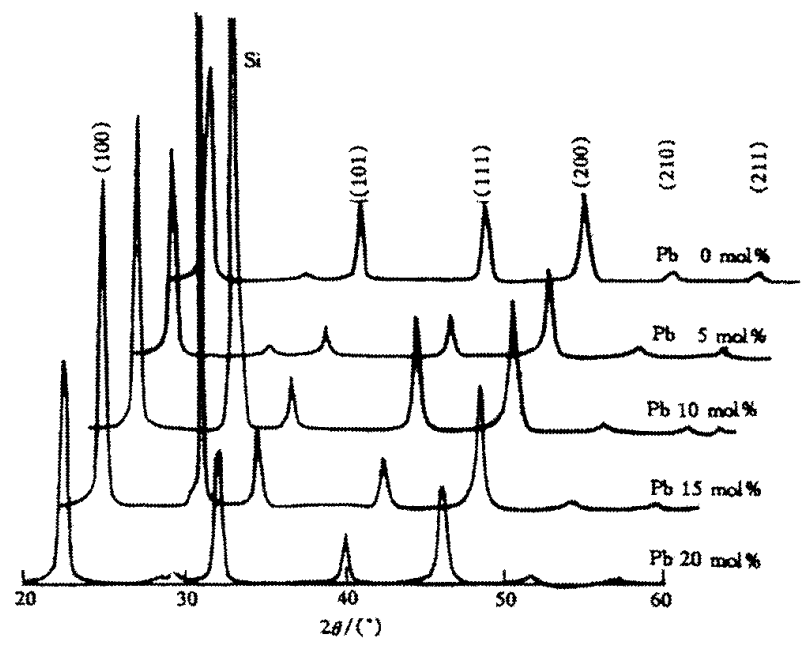

Figure 2. XRD patterns of the PLT10 films with different amounts of excess $\mathrm{PbO}$.

electrode and annealing process. The annealing temperature for all of the film samples was chosen as $550^{\circ} \mathrm{C}$ for $1 \mathrm{~h}$ which was based on the XRD and thermal analysis results. Figure 2 gives the XRD patterns of the PLT10 films with different amounts of excess PbO. It can be seen that these films also had the perovskite structure. Even at high additions of excess $\mathrm{PbO}$, such as $20 \mathrm{~mol} \%$, no $\mathrm{PbO}$ peaks were observed in the XRD patterns. The (100) preferential orientation was found in the PLT films and the intensity of the (100) orientation increased with increasing amounts of excess $\mathrm{PbO}$. The following question is thus asked: has this phenomenon to do with a layer of $\mathrm{PbO}$ formed between the PLT film and bottom electrode? According to the works of Chen and Chen $[16,17]$, the presence of a $\mathrm{PbO}$ buffer layer between the bottom electrode and the PZT film can promote (100) texture formation of the PZT films $[16,17]$. In our case, a layer of $\mathrm{PbO}$ may form between the film and the bottom electrode because of the excess $\mathrm{PbO}$.

The PLT films prepared were crack free and pinhole free, and their surface was very smooth. The AFM photographs of the PLT10 with 20 mol\% excess PbO are shown in figure 3. Crystalline grains could not be distinguished in figure 3(a), and only dense clusters were seen and their scale was about $1 \mu \mathrm{m}$. Further inspection found that the dense clusters were made up of uniform nano-crystals. The grains size of the film is about $50-60 \mathrm{~nm}$.

Figure 4 shows cross section HRTEM images of the PLT thin film with $20 \mathrm{~mol} \%$ excess $\mathrm{PbO}$ deposited on the $\mathrm{Pt} / \mathrm{Ti} / \mathrm{SiO}_{2} / \mathrm{Si}$ substrate. The PLT thin film has a flat surface. The thickness of the PLT films is about $1 \mu \mathrm{m}$. The multilayered structure is clearly observed. The interface between the $\mathrm{Si}$ and the $\mathrm{SiO}_{2}$ layers is very sharp (figure 4(a)). The Ti layer grew in a texture mode (figure 4 (b)). However, the $\mathrm{Ti} / \mathrm{SiO}_{2}$ and $\mathrm{Ti} / \mathrm{Pt}$ interfaces are not sharp. Meanwhile, the interface between the Pt layer and the PLT thin film is not sharp either (figure 4(d)). The Pt layer has an interface because the deposition process was stopped in the middle and then restarted (figure 4(c)).

Figure 4(d) shows that there is transition area between the PLT film and the Pt layer. The transition area has some clusters and the clusters were surrounded by amorphous

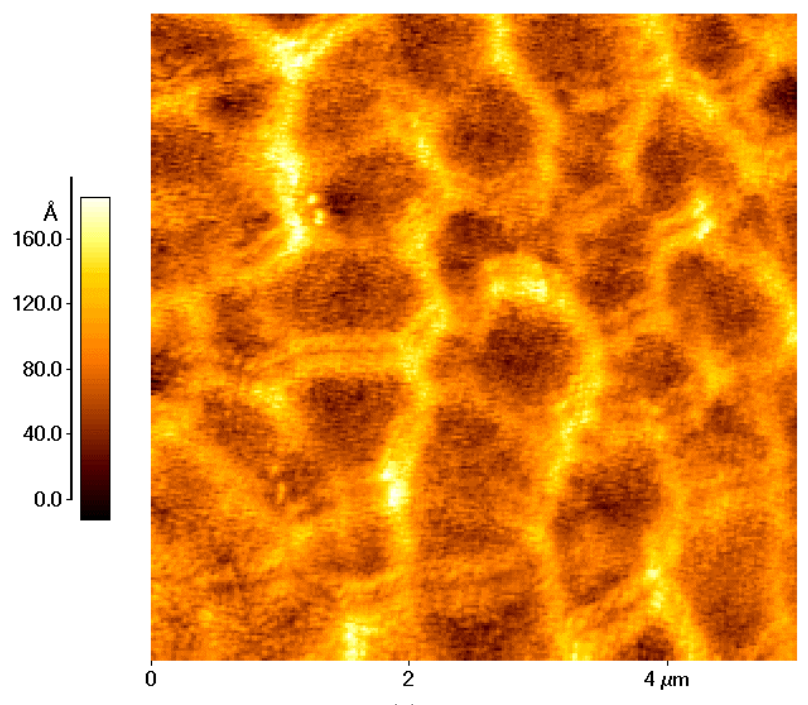

(a)

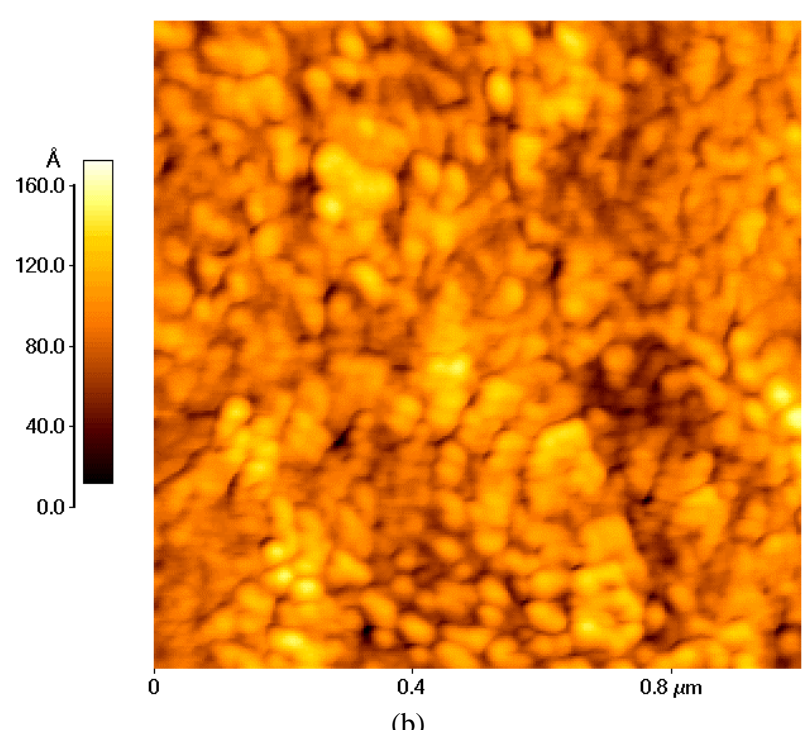

(b)

Figure 3. The AFM photographs of the PLT10 films with $20 \mathrm{~mol} \%$ excess $\mathrm{PbO}$.

atoms. The plane distance and orientation of clusters different from those of the PLT films.

The periodic pattern of lines imaging the atomic planes of the PLT film can readily be seen in figure 4(f), but it is not a prefect crystal. A cluster of interstitials or vacancies can be seen in lattice images. Such clusters can be well described as dislocation rings, and then they should be considered as one- or two-dimensional defects.

\subsection{Composition analysis}

Table 1 shows the EPMA results of the compositions of the PLT10 films with different excess PbO concentrations. The measured $\mathrm{Pb} / \mathrm{Ti}$ ratios for the PLT10 films with higher than $10 \mathrm{~mol} \%$ excess $\mathrm{PbO}$ are larger than 0.923 , which is the calculated ratio without excess $\mathrm{PbO}$ according to the formula in section 2. The measured $\mathrm{Pb} / \mathrm{Ti}$ ratios for the films without excess $\mathrm{PbO}$ are smaller than 0.923 . The above results suggest that after the thermal treatment at $550^{\circ} \mathrm{C}$, the PLT10 films 


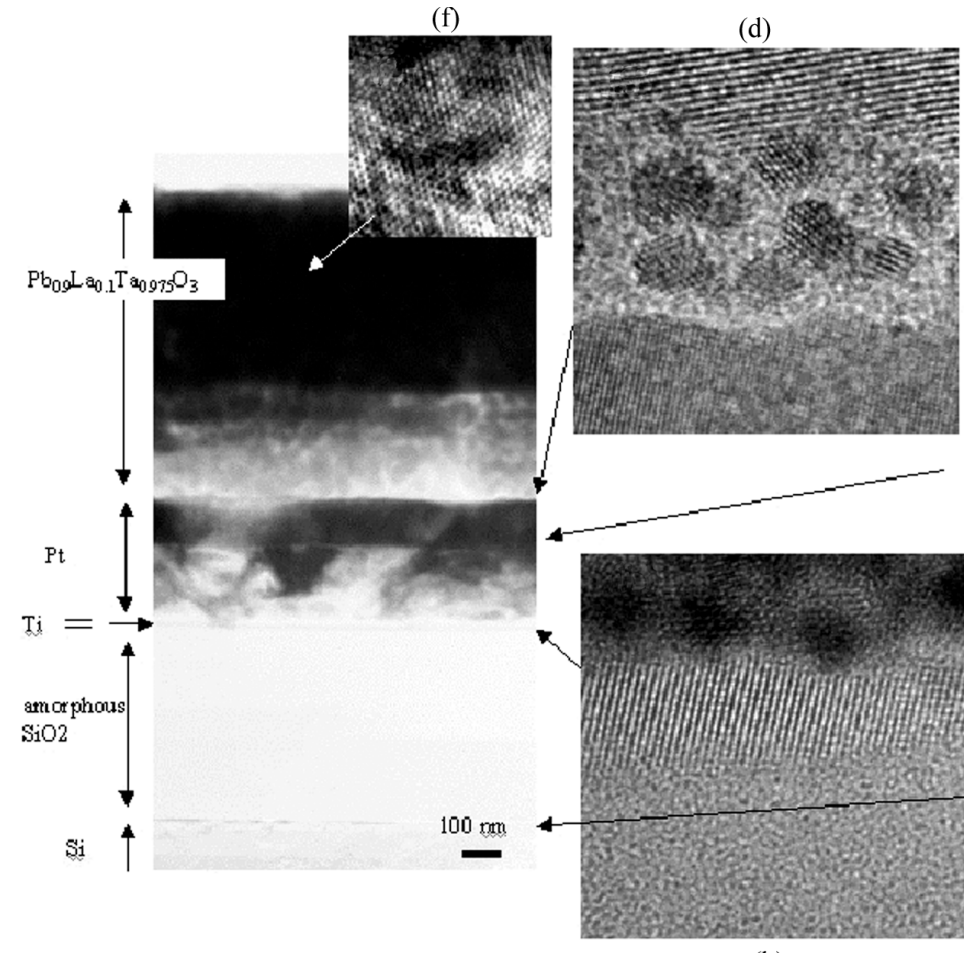

(b) (c)

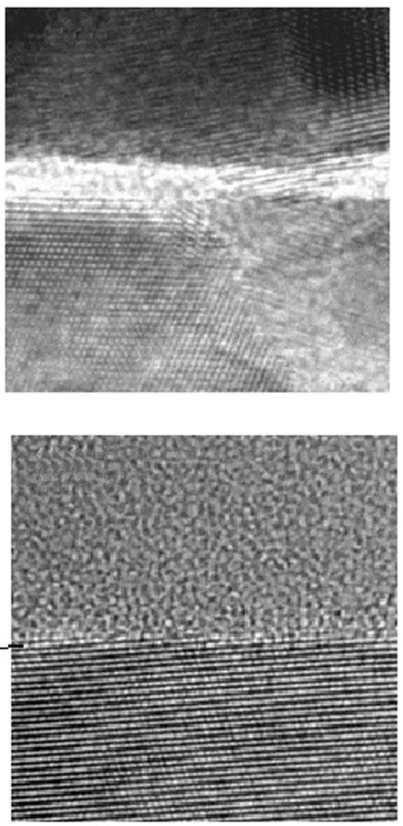

(a)

Figure 4. The HRTEM photographs of the PLT10 film with 20 mol\% excess PbO: (a) the interface of $\mathrm{Si}$ and $\mathrm{SiO}_{2}$, (b) the $\mathrm{SiO} / \mathrm{Ti} / \mathrm{Pt}$ section, (c) the Pt thin film, (d) the transition area between the PLT and the Pt layer, (f) a PLT thin film section.

Table 1. EPMA analysis results of the PLT10 thin films with different amounts of excess Pb.

\begin{tabular}{|c|c|c|c|c|c|c|c|c|c|}
\hline & \multicolumn{3}{|c|}{ at $\%$} & \multicolumn{2}{|c|}{$\mathrm{Pb} / \mathrm{Ti}$} & \multicolumn{2}{|c|}{$\mathrm{La} / \mathrm{Ti}$} & \multicolumn{2}{|c|}{$\mathrm{La}$} \\
\hline & $\mathrm{Pb}$ & $\mathrm{La}$ & $\mathrm{Ti}$ & Original & Measured & Original & Measured & Original & Measured \\
\hline $\mathrm{Pb} 0 \mathrm{~mol} \%$ & 11.82 & 1.38 & 13.47 & 0.923 & 0.878 & 0.103 & 0.105 & 0.100 & 0.102 \\
\hline $\mathrm{Pb} 5 \mathrm{~mol} \%$ & 14.20 & 1.67 & 15.86 & 0.969 & 0.895 & 0.103 & 0.105 & 0.100 & 0.102 \\
\hline $\mathrm{Pb} 10 \mathrm{~mol} \%$ & 12.00 & 1.30 & 12.97 & 1.039 & 0.925 & 0.103 & 0.100 & 0.100 & 0.102 \\
\hline $\mathrm{Pb} 15$ mol\% & 12.10 & 1.27 & 12.95 & 1.015 & 0.934 & 0.103 & 0.101 & 0.100 & 0.099 \\
\hline $\mathrm{Pb} 20$ mol\% & 12.01 & 1.31 & 12.47 & 1.108 & 0.963 & 0.103 & 0.105 & 0.100 & 0.102 \\
\hline
\end{tabular}

Table 2. EPMA analysis results of the PLT10 thin films annealed at different temperatures, with $20 \mathrm{~mol} \%$ excess $\mathrm{PbO}$.

\begin{tabular}{llllll}
\hline $\begin{array}{l}\text { Annealing } \\
\text { temperature } \\
\left({ }^{\circ} \mathrm{C}\right)\end{array}$ & \multicolumn{2}{c}{$\mathrm{Pb} / \mathrm{Ti}$} & & \multicolumn{2}{c}{$\mathrm{La} / \mathrm{Ti}$} \\
\cline { 2 - 3 } \cline { 5 - 6 } & Original & Measured & & Original & Measured \\
\hline 500 & 1.108 & 0.997 & & 0.103 & 0.103 \\
550 & 1.108 & 0.963 & & 0.103 & 0.105 \\
600 & 1.108 & 0.941 & & 0.103 & 0.100 \\
700 & 1.108 & 0.921 & & 0.103 & 0.102 \\
750 & 1.108 & 0.899 & & 0.103 & 0.097 \\
800 & 1.108 & 0.874 & 0.103 & 0.102 \\
\hline
\end{tabular}

with higher than $10 \mathrm{~mol} \%$ excess $\mathrm{PbO}$ still contain excess $\mathrm{Pb}$.

The EPMA results of the compositions of the PLT10 films with $20 \mathrm{~mol} \%$ excess $\mathrm{PbO}$ as a function of the annealing temperature are listed in table 2. The content of $\mathrm{Pb}$ in the films decreases with an increase in the annealing temperature. At annealing temperatures lower than $700{ }^{\circ} \mathrm{C}$, the measured $\mathrm{Pb} / \mathrm{Ti}$ ratios of the PLT10 films with $20 \mathrm{~mol} \%$ excess $\mathrm{PbO}$ are larger than 0.923 .
The AES depth profile of the PLT10 film with $20 \mathrm{~mol} \%$ excess $\mathrm{PbO}$ is plotted in figure 5. We find that there is $\mathrm{Pb}$ accumulation at the interface between the PLT10 film and the Pt electrode. The Pt electrode has a low tolerance for $\mathrm{Pb}$, thus most of the $\mathrm{Pb}$ diffused from the film will go though the Pt electrode into the Si substrate, and only a small part of the $\mathrm{Pb}$ remains in the $\mathrm{Pt}$ electrode. It can be seen from figure 5 that the concentration of $\mathrm{Pb}$ in the PLT10 films varies along thickness direction. This is likely to be related to the inner interfaces formed inside the films by the multi-coating process. It seems that there is $\mathrm{Pb}$ accumulation at the inner interfaces in the PLT10 films.

\section{4. $P-E$ hysteresis loops}

$\mathrm{Au}$ top electrodes with a diameter of $1 \mathrm{~mm}$ were sputtered onto the surface the samples by a shadow mask. A sandwich structure of Au/PLT/Pt was formed and used to measure the electrical properties.

Figure 6 shows the $P-E$ hysteresis loops of the PLT10 films with different amounts of excess $\mathrm{PbO}(0-20 \mathrm{~mol} \%)$. The sine-wave voltage applied was $30 \mathrm{~V}$, peak-peak. At this 


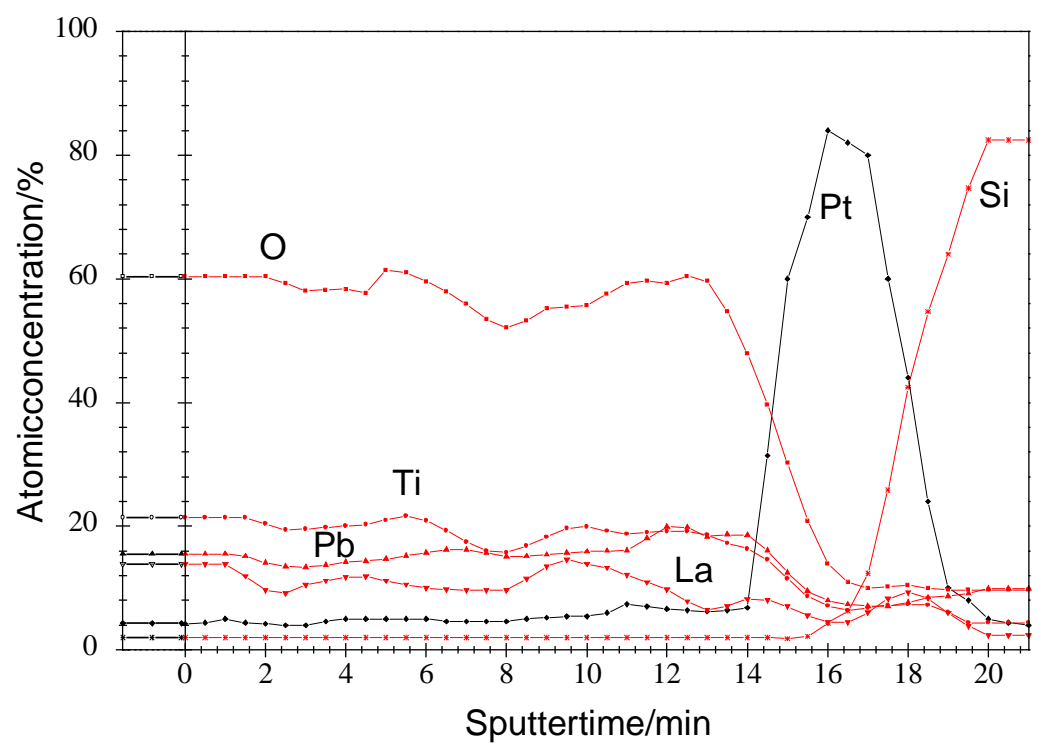

Figure 5. AES depth profiles of the PLT10 thin film with $20 \mathrm{~mol} \%$ excess PbO

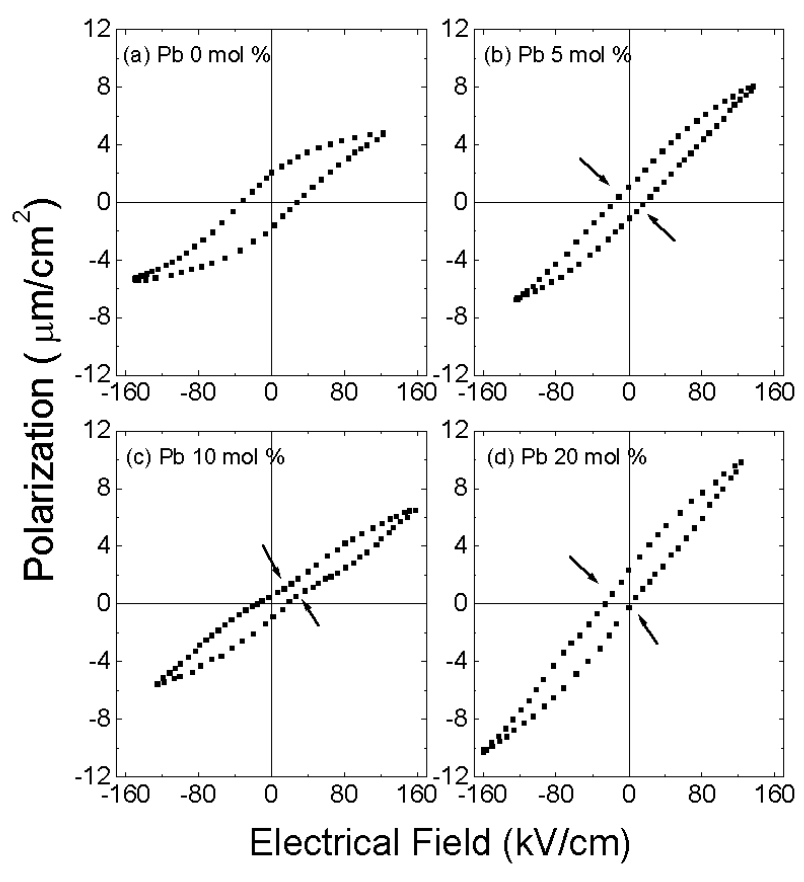

Figure 6. The $P-E$ hysteresis loops of the PLT10 films with different amounts of excess $\mathrm{PbO}$.

voltage, the films are not saturated. In figure 6(a) it can be seen that the normal hysteresis loop was observed in the PLT10 films with no excess $\mathrm{PbO}$, but after adding excess $\mathrm{PbO}$, the abnormal hysteresis loops appeared in the PLT films and these loops had a calabash-shape, which means that the loops became narrow in the middle part. The abnormal level was more severe with increased excess amounts of $\mathrm{PbO}$, as seen in figures 6(b)-6(d).

The abnormal hysteresis loops can turn to normal hysteresis loops by applying a higher poling voltage. Figure 7 is the hysteresis loops of the PLT10 films with $10 \mathrm{~mol} \%$ excess $\mathrm{PbO}$ at different voltages. The abnormal level is decreased with an increase of the voltage. The loop became

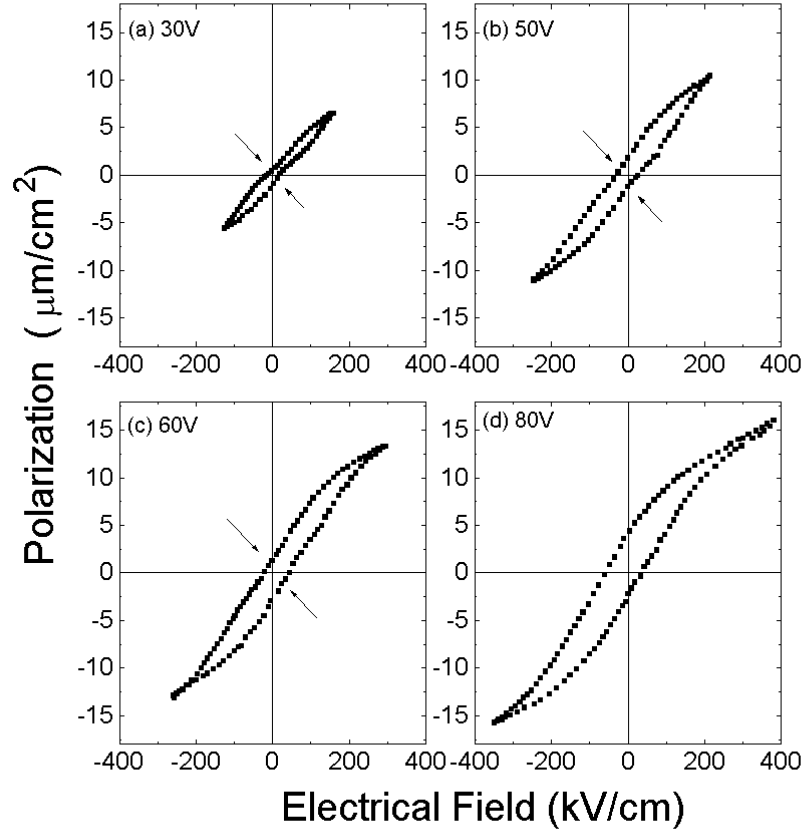

Figure 7. $P-E$ hysteresis loops of the PLT10 films with $10 \mathrm{~mol} \%$ excess $\mathrm{PbO}$ at different voltages.

normal and was saturated at a voltage of $80 \mathrm{~V}$ peak-peak, as shown in figure $7(d)$.

The above results reveal that the abnormal hysteresis loops appeared in all of the PLT thin films with excess $\mathrm{PbO}$ and this abnormality can be overcome by applying a higher poling voltage.

\section{5. $C-V$ characteristics}

For the $C-V$ curve measurement by a HP 4192A LP impedance analyser, a $0.5 \mathrm{~V}$ sine-wave voltage at a frequency of $10 \mathrm{kHz}$ was overlapped on a dc bias voltage which scanned linearly from $-20 \mathrm{~V}$ to $20 \mathrm{~V}$ and vice versa at a step of $0.5 \mathrm{~V}$ and a speed of $0.5 \mathrm{~V} \mathrm{~s}^{-1}$. Figure 8 shows the $C-V$ 


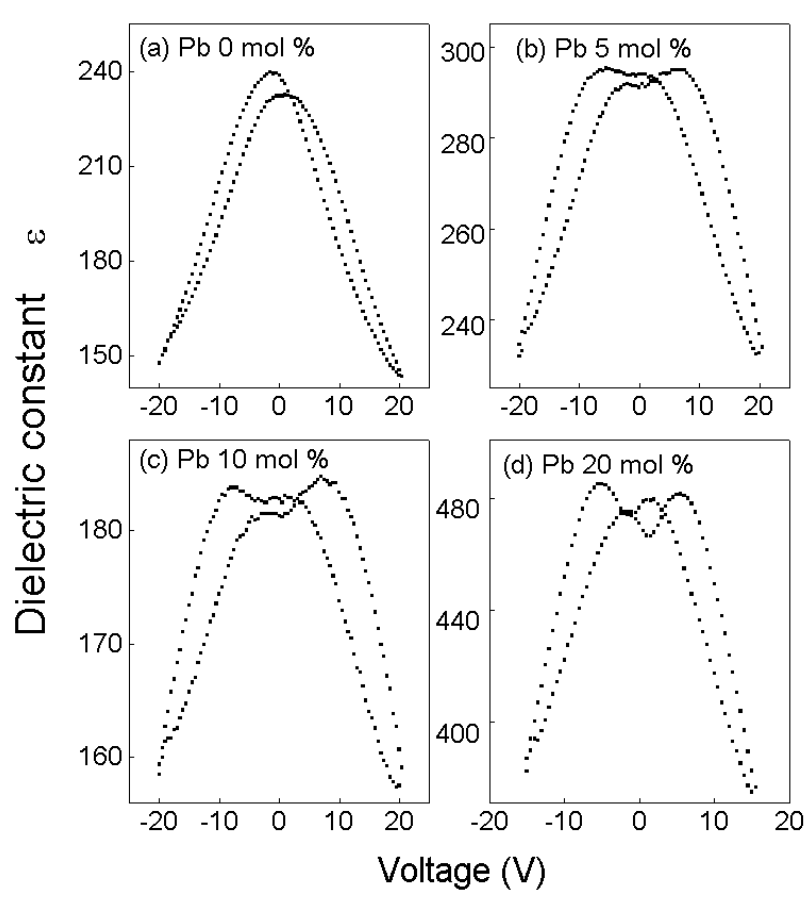

Figure 8. $C-V$ curves of the PLT10 films with different amounts of excess $\mathrm{PbO}$.

curves of the PLT10 films with different amounts of excess $\mathrm{PbO}$. The $C-V$ curve of the PLT10 with no excess PbO was a normal two-peak curve (butterfly shape) as shown in figure 8(a). The values of the bias voltages for the two peaks were nearly equal. The different heights of the peaks may be attributed to the different interfaces of the top and bottom electrodes with the films. For the PLT10 films with excess $\mathrm{PbO}$, the abnormal four peaks instead of the two peaks (as in normal case) were observed in the $C-V$ curves, which are shown in figures $8(\mathrm{~b})-8(\mathrm{~d})$. In the positive scanning mode (from $-20 \mathrm{~V}$ to $20 \mathrm{~V}$ ), a small peak appeared before the bias voltage reached zero and the second large peak appeared at a positive bias voltage. The height of the second peak was larger than that of the first peak. In the negative scanning mode (from $20 \mathrm{~V}$ to $-20 \mathrm{~V}$ ), two similar peaks were also found. The abnormality of $C-V$ curves was more severe with increased amounts of excess $\mathrm{PbO}$.

Measurement conditions such as frequency and bias voltage also had strong effect on the abnormality of the $C-V$ curves. Figure 9 gives the $C-V$ curves of the PLT10 films with $20 \mathrm{~mol} \%$ excess $\mathrm{PbO}$ at different scanning ranges of the bias voltage. At the scanning range of $\pm 5 \mathrm{~V}$, the $C-V$ curve was abnormal, with distinct four peaks (figure 9(a)). The degree of abnormality decreased with an increase of the scanning range of the bias voltage. The normal $C-V$ curve with two peaks was observed at the scanning range of $\pm 25 \mathrm{~V}$ (figure 9(b)). Figure 10 shows the $C-V$ curves of the PLT10 films with $20 \mathrm{~mol} \%$ excess $\mathrm{PbO}$ at different frequencies. The $C-V$ curve was abnormal at a frequency of $10 \mathrm{kHz}$, as shown in figure 10(a) and can be changed to a normal curve by increasing of the frequency (as shown in figure 10(b) at a frequency of $100 \mathrm{kHz}$ ).

Summarizing the measured results of the $C-V$ characteristics, we may get following facts: the abnormal

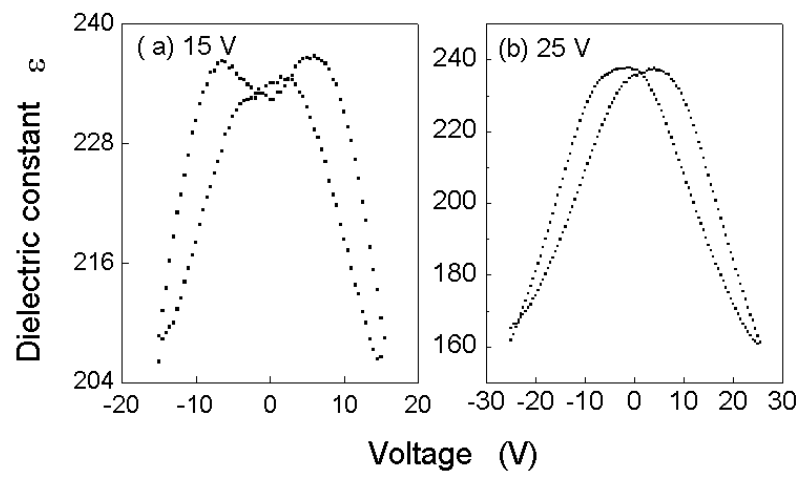

Figure 9. $C-V$ curves of the PLT films with $20 \mathrm{~mol} \%$ excess $\mathrm{PbO}$ at different scanning ranges of the bias voltages.

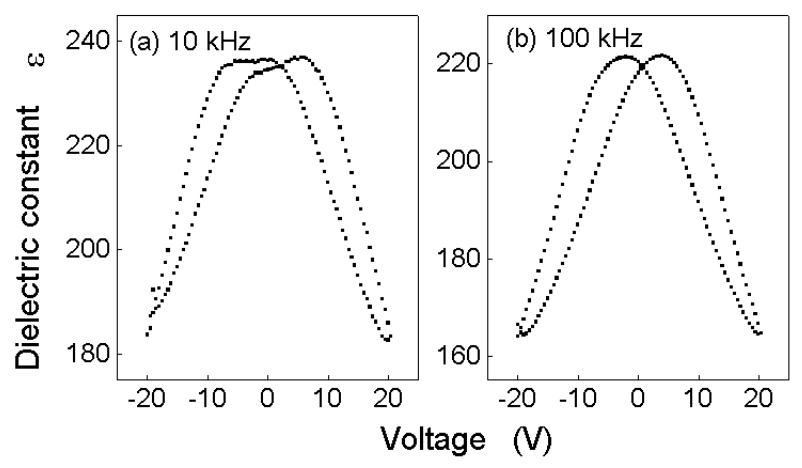

Figure 10. $C-V$ curves of the PLT films with $20 \mathrm{~mol} \%$ excess $\mathrm{PbO}$ at different frequencies.

$C-V$ curves with four peaks in the PLT films were caused by the addition of excess $\mathrm{PbO}$. The degree of abnormality of the $C-V$ curves was more severe with an increase in the amount of excess $\mathrm{PbO}$. These curves can be transformed into the normal curves by increasing of the frequency of the sine-wave whilst keeping the amplitude constant or/and by increasing of the scanning ranges of the bias voltages at a given frequency.

\section{Discussions}

\subsection{Interrelation between abnormal $P-E$ hysteresis loops and abnormal $C-V$ curves}

The basic conclusions we obtained from the section 3 about abnormal $P-E$ hysteresis loops and abnormal $C-V$ curves are one-to-one correspondence. They have close interrelationship and are the reflection from two different aspects about the reversal process of the ferroelectric domains in the $\mathrm{PT}$ and PLT films with excess PbO under an electric field that is below the normal poling field.

Figure 11 gives an example a normal $C-V$ curve and $P-E$ hysteresis loop of the PLT films with no excess PbO. The $P-E$ hysteresis loop indicates that the relationship between the polarization $\boldsymbol{P}$ and the electric field $\boldsymbol{E}$ is nonlinear. From $\boldsymbol{D}=\varepsilon_{0} \boldsymbol{E}+\boldsymbol{P}$, we have $\varepsilon=\mathrm{d} \boldsymbol{D} / \mathrm{d} \boldsymbol{E}=$ $\varepsilon_{0}+\mathrm{d} \boldsymbol{P} / \mathrm{d} \boldsymbol{E} \approx \mathrm{d} \boldsymbol{P} / \mathrm{d} \boldsymbol{E}$ (the last equation is valid for the PLT films). In our $C-V$ measurement, a sine-wave voltage of $0.05 \mathrm{~V}$ and $10 \mathrm{kHz}$ was overlapped on a dc bias voltage of $\pm 20 \mathrm{~V}$ and the measured dielectric constants are reversible values. The reversible dielectric constants are 


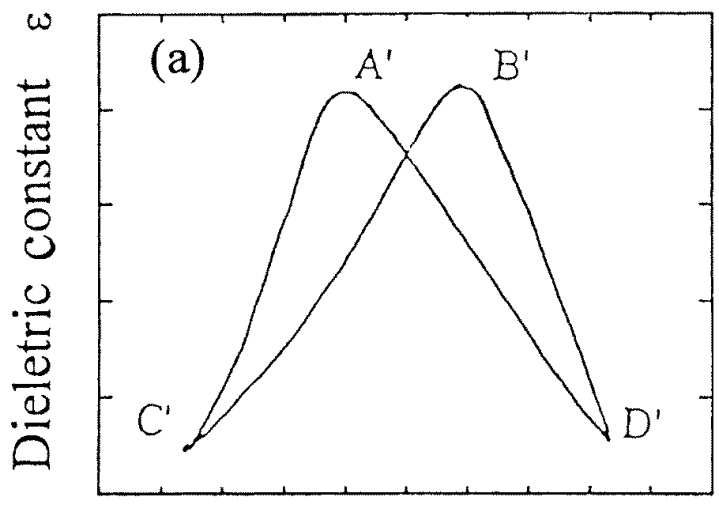

Applied DC Field (KV/cm)

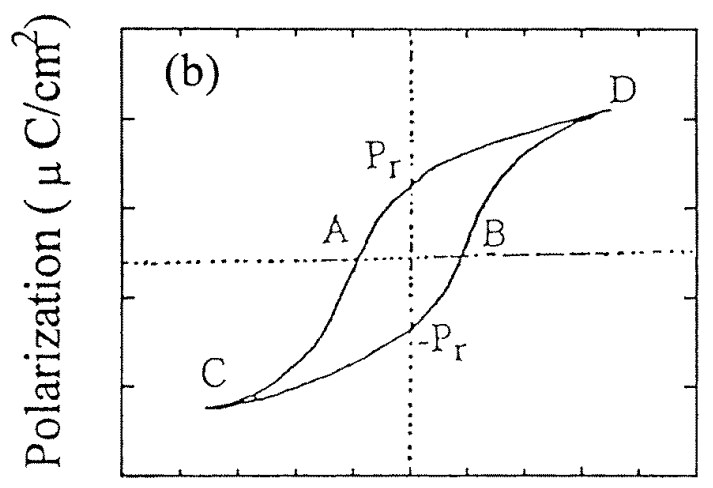

Applied Electric Field $(\mathrm{KV} / \mathrm{cm})$

Figure 11. The normal $P-E$ hysteresis loops and $C-V$ curves of the PLT films with no excess PbO.

equal to or smaller than the differential dielectric constants at the same electric field, and the differential dielectric constants of ferroelectric materials are equal to the slope of the $P-E$ hysteresis loop at the same electric field. Because of the small effect of the non-reversible factor on the PLT films, the $C-V$ dielectric constants measured are approximately equal to the dielectric constants from the $P-E$ hysteresis loop at the corresponding field.

The relationship between the $P-E$ hysteresis loop and the $C-V$ curve of the PLT films can also been explained by the reversal process of the domains.

The $P-E$ hysteresis loop gives the polarization of the ferroelectric films as a function of the electric field. For the films as deposited by the MOD process, the orientation of the domains is usually random. Under the applied field, reorientation of the domains mostly occurs around the coercive field of the films and leads to the largest change of the polarization. The $\mathrm{A}$ and $\mathrm{B}$ points in the $P-E$ loop in figure 11(a) correspond to the $\mathrm{A}^{\prime}$ and $\mathrm{B}^{\prime}$ points in the $C-V$ curve in figure $11(\mathrm{~b})$, and the two points $\mathrm{A}^{\prime}$ and $\mathrm{B}^{\prime}$ correspond to the maximum dielectric constants. At the points $\mathrm{C}$ and $\mathrm{D}$ in the $P-E$ loop, most domains have been re-orientated under the electric field and there is little change of the polarization, so these two points are correspondent to the points $\mathrm{C}^{\prime}$ and $\mathrm{D}^{\prime}$ in the $C-V$ curve.
From the point view of mathematics, the line segment $\mathrm{CBD}$ in the $P-E$ loop corresponds to the line segment $\mathrm{C}^{\prime} \mathrm{B}^{\prime} \mathrm{D}^{\prime}$ in the $C-V$ curve. The points $\mathrm{A}^{\prime}$ and $\mathrm{B}^{\prime}$ are maxims of the $\varepsilon$. Because of $\varepsilon \approx \mathrm{d} P / \mathrm{d} E$, we have $\mathrm{d} \varepsilon / \mathrm{d} E=\mathrm{d}^{2} P / \mathrm{d} E^{2}=0$, so the points $\mathrm{A}$ and $\mathrm{B}$ are the two knees of the function $P=P(E)$. In the segment CBD, there are $\mathrm{d}^{2} P / \mathrm{d} E^{2}>0$ in the segment $\mathrm{CB}$ and $\mathrm{d}^{2} P / \mathrm{d} E^{2}<0$ in the segment BD. In the segment DAC, there are $\mathrm{d}^{2} P / \mathrm{d} E^{2}<0$ in the segment DA and $\mathrm{d}^{2} P / \mathrm{d} E^{2}>0$ in the segment AC.

Figure 12(a) shows the abnormal $C-V$ curve of the PLT films with excess $\mathrm{PbO}$. There are two peaks $\left(\mathrm{B}^{\prime}\right.$ and $\left.\mathrm{C}^{\prime}\right)$ and one trough $\left(\mathrm{D}^{\prime}\right)$ in the positive scanning process from point $\mathrm{A}^{\prime}$ to point $\mathrm{E}^{\prime}$, which means that there should be three extremities. At the points $\mathrm{B}^{\prime}, \mathrm{C}^{\prime}$ and $\mathrm{D}^{\prime}$, we have $\mathrm{d} \varepsilon / \mathrm{d} E=\mathrm{d}^{2} P / \mathrm{d} E^{2}=0$. Based on the above analysis, there should be three knees in the poling process from point A to point $\mathrm{E}$ of the $P-E$ hysteresis loop in figure 12(b). As the points $\mathrm{B}^{\prime}$ and $\mathrm{D}^{\prime}$ are maxima, and the point $\mathrm{C}^{\prime}$ is a minimum, $\mathrm{d} \varepsilon / \mathrm{d} E=\mathrm{d}^{2} P / \mathrm{d} E^{2}>0$ in the segments $\mathrm{AB}$ and $\mathrm{CD}$ and $\mathrm{d} \varepsilon / \mathrm{d} E=\mathrm{d}^{2} P / \mathrm{d} E^{2}<0$ in the segments BC and $\mathrm{DE}$. So the segments $\mathrm{AB}$ and $\mathrm{CD}$ are concave-up and the segments $\mathrm{BC}$ and $\mathrm{DE}$ are convex. A similar analysis can be used for the negative scanning process from point $\mathrm{E}^{\prime}$ to point $\mathrm{A}^{\prime}$ in the $C-V$ curve. The $P-E$ hysteresis loop can be drawn according to above analysis, which is shown in figure 12(b). This curve is very similar to the $P-E$ curve of the PLT10 films with excess PbO, as shown in figure 8 . Now we have proved from mathematical analysis that the abnormal $C-V$ curve with four peaks is consistent with the abnormal calabash shape of the $P-E$ hysteresis loop.

\subsection{The pinning effect as an explanation of the abnormality}

The abnormal $P-E$ hysteresis loops and $C-V$ curves of the $\mathrm{PT}$ and PLT films with excess $\mathrm{PbO}$ can be explained by ferroelectric pinning.

For the PLT films with no excess $\mathrm{Pb}$, the defects in the grain boundaries and the interfaces between the film and electrode do not result in the above abnormality. The abnormality only appears in films with excess $\mathrm{PbO}$ and may be the caused by the pinning effect on the ferroelectric domains by the $\mathrm{PbO}$ phases formed by the excess $\mathrm{Pb}$ on the domain wall, grain and cluster boundaries and interfaces, which act as pinning centres.

The composition analysis shows that when the annealing temperature is $550{ }^{\circ} \mathrm{C}$, the measured $\mathrm{Pb} / \mathrm{Ti}$ ratio in PLT10 films with higher than $10 \mathrm{~mol} \%$ excess $\mathrm{PbO}$ is larger than 0.923; when the annealing temperature is lower than $700{ }^{\circ} \mathrm{C}$, the measured $\mathrm{Pb} / \mathrm{Ti}$ ratio in PLT10 films with $20 \mathrm{~mol} \%$ excess $\mathrm{PbO}$ is larger than 0.923 . This means that these films still contain excess $\mathrm{Pb}$.

According to the results of AES depth profile analysis, there is $\mathrm{Pb}$ accumulation at the film/bottom electrode interface and the HRTEM image shows that there is a transition area at the film/bottom electrode interface where some clusters are found. These clusters may be $\mathrm{PbO}$ spheres.

The cluster of interstitials or vacancies in crystalline grains of the PLT film with $20 \mathrm{~mol} \%$ excess $\mathrm{PbO}$ can be seen in lattice images. The cluster of interstitials may be caused 


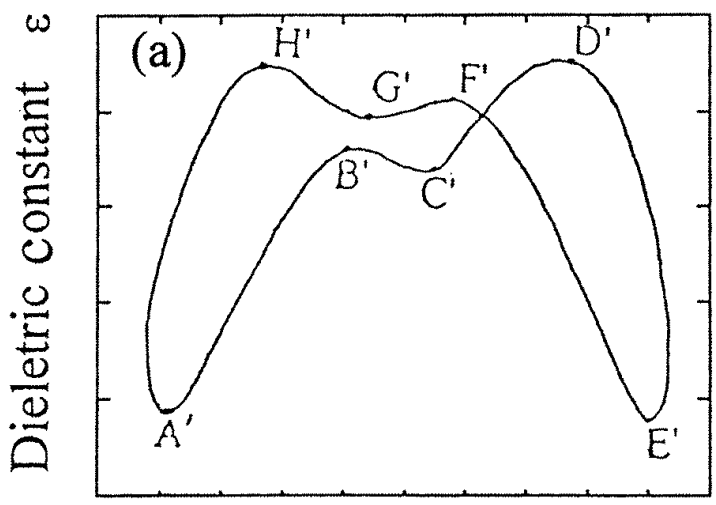

Applied DC Field $(\mathrm{KV} / \mathrm{cm})$

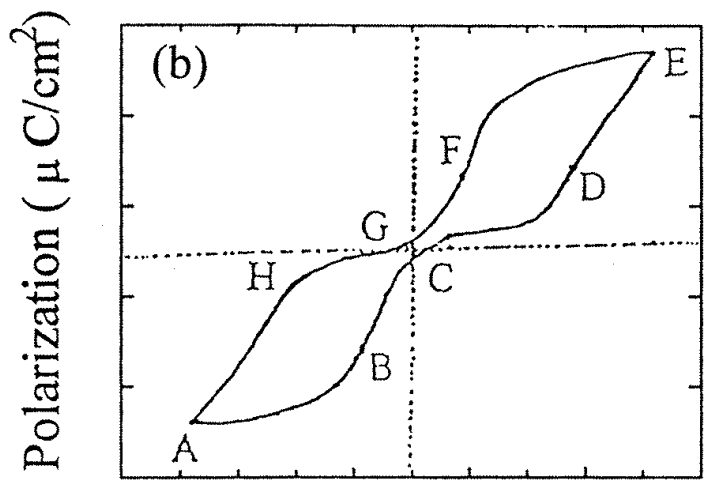

Applied Electric Field $(\mathrm{KV} / \mathrm{cm})$

Figure 12. The abnormal $P-E$ hysteresis loops and $C-V$ curves of PLT10 with 10 mol\% excess PbO.

by excess $\mathrm{Pb}$ in the films. The cluster of vacancies may be related to oxygen vacancies. Oxygen vacancies usually exist in the perovskite structure films. For PLT thin films with excess $\mathrm{PbO}$, a group of interstitial $\mathrm{Pb}$ ions may absorb oxygen ions at some normal lattice sites to form $\mathrm{PbO}$ due to electric neutrality, and thus result in the greater number of oxygen vacancies in the PLT films with excess $\mathrm{PbO}$ than in the PLT film with less PbO.

After slight modification, the model of the nucleation and growth of the perovskite PZT films can be used to describe the formation process of the $\mathrm{PbO}$ phase as pinning centres in the PLT films with excess PbO [14]. The nucleation and growth of perovskite phase occurrs directly during the thermal process in the PLT films with no excess $\mathrm{Pb}$, as shown in figure 13(a). While for the PLT films with excess $\mathrm{PbO}$, because the perovskite phase has a limited tolerance for excess $\mathrm{Pb}$ ions (usually within $1-2 \mathrm{~mol} \% \mathrm{~Pb}$ tolerance) [14], excess $\mathrm{Pb}$ ions would be rejected out by the perovskite phase during the nucleation and growth process which is illustrated in figure 13(b). According to the thermal analysis in section 3.1 and the composition analysis in the section 3.3, there was no obvious $\mathrm{Pb}$ loss when the annealing temperature was lower than $600^{\circ} \mathrm{C}$, and a large part of the excess $\mathrm{Pb}$ still remains in the PLT films. Some of the excess $\mathrm{Pb}$ emerged in the grains in form of small clusters, which consist of

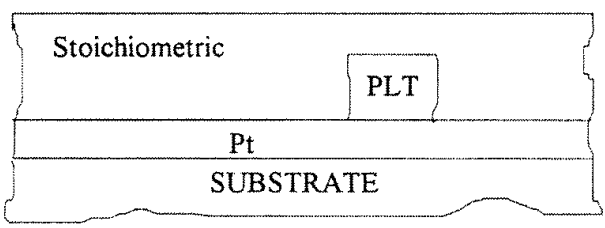

(a)

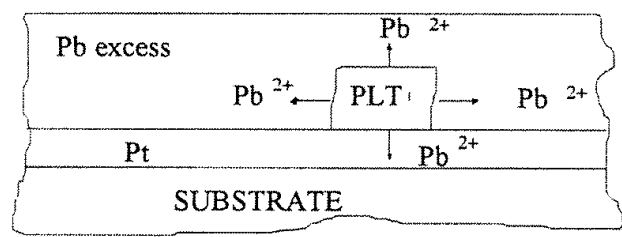

(b)

Figure 13. Schematic diagram of the nucleation and growth model for (a) stoichiometric PLT films and (b) PLT films with excess $\mathrm{Pb}$.

interstitials because the perovskite phase has a tolerance for the $\mathrm{Pb}$ ions. Meanwhile, the clusters of interstitials may cause more oxygen vacancies in the PLT grains. Another part of it emerged in the inerface of grains and clusters in form of $\mathrm{PbO}$ phase. The rest of the excess $\mathrm{Pb}$ possibly diffuses into the interface between the film and the bottom electrode to form a $\mathrm{PbO}$ layer, which also has pinning effects on the domains. The pinning effect of the $\mathrm{PbO}$ phase and vacancies resulted in the abnormality of the $P-E$ hysteresis loops and $C-V$ curves of the PLT thin films with excess PbO.

\subsection{Explanation experimental results}

$\mathrm{Pb}$ ions remain in the grains and diffuse to the boundaries and interfaces during the nucleation and growth of the perovskite grains in the PLT films. The $\mathrm{Pb}$ ions which diffuse to the interface between the film and the bottom electrode may stay in the form of the $\mathrm{PbO}$ phase, while the $\mathrm{PbO}$ ions which diffuse to the surface of the films may evaporate. The concentration of the $\mathrm{PbO}$ layer in the interface between the film and the bottom electrode was greater than that between the film and the upper electrode, and this resulted in the different pinning forces at the bottom and upper interfaces. So that the $P-E$ loops had a shift at the electric field axis and the areas of the upper part and lower part of the calabashshaped $P-E$ loop were not same.

According to Coulomb's law, with an increase of the distances from the domain wall, the crystalline grain boundaries, cluster boundaries and the interfaces between the film and the electrode, the Coulomb force to the ferroelectric domains decreases.

With increasing amounts of $\mathrm{PbO}$, the clusters, interstitials or vacancies of grains and $\mathrm{PbO}$ phase of the grain boundaries may increase and the pinning effect on the ferroelectric domains also increases. So that we observed the abnormal level of the $P-E$ loops and $C-V$ curves increased with increasing amounts of excess $\mathrm{PbO}$.

The abnormal $P-E$ hysteresis loop can be changed to the normal-type loop with an increase of the poling voltage and the $C-V$ curves can be changed from abnormal four peaks to normal two peaks with an increase of the bias voltage. 
All of these effects are due to the fact that the pinning force has a limited strength. When the applied field is larger than the field produced by the $\mathrm{PbO}$ and oxygen vacancies pinning centres, the reversal process of the domains can be carried out normally and the polarization process of the films develops into a stable state from a metastable state.

When the poling voltage for the $P-E$ loops and the bias voltage for the $C-V$ curves are held constant, the reversal probability of the domains is increased with an increase of the bias frequency. This could shake the pinning effect on the domains by the $\mathrm{PbO}$, and the domains which were not reversible at low frequency will reverse at a higher frequency. The abnormality of both the $P-E$ loops and $C-V$ curves becomes weaker with an increase of frequency, and the abnormal $P-E$ loops and $C-V$ curves can even change to the normal loops and curves, as shown in figure 10(b).

\section{Conclusions}

The PLT thin films with excess $\mathrm{PbO}$ were prepared by the MOD process. The $C-V$ characteristics and ferroelectric properties of the PLT films were investigated as a function of the amount of excess of the $\mathrm{PbO}$. Normal ferroelectric hysteresis loops and butterfly-shaped $C-V$ curves were obtained for the PLT films with stoichiometric composition. Abnormal ferroelectric and $C-V$ properties were observed in the PLT films with excess PbO. The abnormal level of the hysteresis loops and $C-V$ curves were more severe for larger amounts of excess $\mathrm{PbO}$ in the films. The experimental results can be explained by the ferroelectric pinning effect. Some excess $\mathrm{PbO}$ may accumulate at the domain walls of grains and the boundaries of crystalline grains and clusters and the interfaces between the bottom electrode and the film during the annealing process of the PLT films. Meanwhile, oxygen vacancies of the grains may be increased with excess $\mathrm{PbO}$. This part of the $\mathrm{PbO}$ and oxygen vacancies of the grains acted as the pinning centres and had a strong effect on the ferroelectric domains. When the poling voltage is not large enough, some of the domains cannot overcome the force of the pinning, and abnormal ferroelectric and $C-V$ properties are observed. These and other results concerning different La content and SEM observation, in particular, the physical mechanism of the domains pinning caused by excess $\mathrm{PbO}$, will be published in a more detailed paper.

\section{Acknowledgments}

This work is supported by the National Advanced Materials Committee of China (NAMCC), No 715-002-0110 and the National Science Nature Foundation of China, No 59902012 and No 69738020.

\section{References}

[1] Dey S K and Lee J J 1992 IEEE Trans. Electron. Devices 39 1607

[2] Takayama R, Tomita Y, Iijima K and Ueda I 1987 J. Appl. Phys. 61411

[3] Ren W, Liu Y, Wu X Q, Zhang L Y and Yao X 1997 Integrated Ferroelectrics $\mathbf{1 5} 271$

[4] Adachi H, Mitsuyu T, Yamazaki O and Wasa K 1986 J. Appl. Phys. 60736

[5] Yoshioka H 1994 J. Mater. Res. 92133

[6] Schwartz R W, Tuttle B A, Doughty D H, Land C E, Goodnow D C, Hernandez C L, Zender T J and Martinez S L 1991 IEEE Trans. Ultrasonics, Ferroelectrics Frequency Control 38677

[7] Ren W, Liu Y, Qiu J H, Zhang L Y and Yao X 1994 Ferroelectrics 152201

Liu Y, Ren W, Qiu J H, Zhang L Y and Yao X 1994 Ferroelectrics 152195

[8] Iijima K, Takayama R, Tomita Y and Ueda I 1986 J. Appl. Phys. 602914

[9] Wang J P, Ling Y C, Yeh M H, Liu K S and Lin I N 1996 Appl. Phys. Lett. 683401

[10] Tuttle B A, Schwartz R W, Doughty D H, and Voigt J A 1990 Ferroelectric Thin Films (Material Research Society Symposium Proceedings vol 200) ed E R Myers and A I Kingon (Pittsburgh, PA: Material Research Society) p 159

[11] Bernstein S D, Kisler Y, Wahl J M, Bernacki S E and Collins S R 1992 Ferroelectric Thin Films II (Material Research Society Symposium Proceedings vol 200) ed A I Kingon, E R Myers and B Tuttle (Pittsburgh, PA: Material Research Society) p 373

[12] Bernstein D, Wong T Y, Collins S R, Kisler Y and Tustison R W 1995 Ferroelectric Thin Films IV (Material Research Society Symposium Proceedings vol 200) ed B A Tuttle, S B Desu, R Ramesh and T Shiosaki (Pittsburgh, PA: Material Research Society) p 477

[13] Khan A R and Desu S B 1995 J. Mater. Res. 102777

[14] Lefevre M J, Speck J S, Schwartz R W, Doimos D and Lockwood 1996 J. Mater. Res. 112076

[15] Kim T S, Kim D J, Lee J K and Jung H J 1998 J. Mater. Res. 133436

[16] Chen S Y and Chen I W 1994 J. Am. Ceram. Soc. 772332

[17] Chen S Y and Chen I W 1994 J. Am. Ceram. Soc. 772337 